\title{
Stopping Empagliflozin Unmasks Heart Failure
}

\author{
Morolake Amole, MD; and Julio Leey-Casella, MD
}

SGLT2 inhibitors have been shown to have a role in the management of heart failure in
patients with type 2 diabetes mellitus, but there is a risk of exacerbation when discontinued.

Morolake Amole is an
Endocrinology Fellow,
and Julio Leey-Casella
is an Assistant Professor
of Medicine and
Endocrinology, both at
the University of Florida
College of Medicine. Julio
Leey-Casella is an Endocri-
nologist at Malcolm Randall
Veterans Affairs Medical
Center in Gainesville.
Correspondence:
Morolake Amole
(mamole001@gmail.com)

Fed Pract. 2021;38(6).

Published online June 17. doi:10.12788/fp.0144
A bout $40 \%$ of patients with heart failure (HF) also have type 2 diabetes mellitus (T2DM). ${ }^{1}$ Certain sodiumglucose cotransporter-2 (SGLT2) inhibitors have benefited patients with $\mathrm{HF}^{2}$ We report a case of a patient with T2DM who had signs and symptoms of hypervolemia after discontinuing the SGLT2 inhibitor empagliflozin. The patient was found to have previously undiagnosed HF. This case highlights the relationship between T2DM and $\mathrm{HF}$ and the benefits of treatment with SGLT2 inhibitors.

\section{CASE PRESENTATION}

A 58-year-old male presented for care at Malcolm Randall Veterans Affairs Medical Center in Gainseville, Florida, diabetes clinic. The patient was diagnosed with T2DM at age 32 years. At 36 years, he was started on subcutaneous insulin injections, and was switched to insulin pump therapy in his early 40s. At the time of evaluation, the T2DM was managed using an insulin pump, metformin, and acarbose. He had been prescribed empagliflozin $10 \mathrm{mg} \mathrm{sev-}$ eral months before presentation, but the medication ran out about 1 month prior to evaluation, and additional refills were unavailable.

The patient reported a 1-month history of worsening exertional shortness of breath, decreased exercise tolerance, and lower extremity swelling. Vitals signs, including respiratory rate and oxygen saturation were within normal limits. Bibasilar crackles and bilateral 2+ pitting pedal edema were noted. The remaining examination was unrevealing. His most recent glycated hemoglobin $A_{1 c}$ level from 1 month prior to the presentation was $6.4 \%$.
Given the patient's shortness of breath and evidence of fluid overload on examination, brain natriuretic peptide was obtained and was significantly elevated at $5,895 \mathrm{pg} / \mathrm{mL}$. A transthoracic echocardiogram revealed left ventricular ejection fraction $<20 \%$. The patient was started on furosemide $40 \mathrm{mg}$, pending receipt of empagliflozin. A cardiology evaluation also was recommended.

Cardiac catheterization identified significant obstructions to the left anterior descending and left circumflex arteries. The patient underwent successful percutaneous coronary intervention to these areas. Following initiation of medications and coronary revascularization, the patient reported significant symptom improvement. At the follow-up evaluation 8 weeks later, he was symptom free, and his physical examination was consistent with euvolemia.

\section{DISCUSSION}

T2DM has been associated with adverse cardiovascular outcomes, including atherosclerotic heart disease and HF. There are several theories about the relationship between T2DM and HF, though the exact pathophysiology of this relationship is unknown. ${ }^{3,4}$ One theory suggests diabetic cardiomyopathy as the cause. In patients with diabetic cardiomyopathy, there is early development of diastolic dysfunction, which eventually progresses to ventricular dysfunction. There is continued stimulation of the reninangiotensin-aldosterone system that leads to death of cardiomyocytes, fibrosis, and remodeling, which worsens pump failure. ${ }^{5}$

SGLT2 inhibitors decrease hyperglycemia and hyperinsulinemia, potentially reducing HF risk. SGLT2 inhibitors decrease blood glucose levels by inhibiting SGLT2 in 
the proximal tubule, leading to a decrease of glucose reabsorption and an increase in excretion. ${ }^{6,7}$ The EMPA-REG OUTCOME trial looked at cardiovascular outcomes in patients with T2DM at high risk for adverse cardiac events. There was a significant risk reduction in deaths and hospitalizations for HF in patients treated with empagliflozin. ${ }^{8}$

The EMPRISE study specifically examined empagliflozin and its effects on hospitalization for $\mathrm{HF}^{2}$ When compared with patients treated with sitagliptin, there was a statistically significant decrease in hospitalization for HF in patients with T2DM, both with and without preexisting cardiovascular disease.

This case highlights the relationship between T2DM and HF. We also show how the use of empagliflozin may have helped manage the patient's undiagnosed HF and how its discontinuation luckily unmasked it. Routine evaluation for HF in patients with T2DM is not done, but likely there are patients who would benefit, especially given the strong, albeit less known, association between these 2 conditions.

Further studies are needed to determine the type of patients who would benefit most from HF screening. For now, the best practice is to obtain a complete medical history that includes current and recently discontinued medications as well a thorough physical examination for signs of fluid overload and cardiovascular compromise. Patients who may have signs concerning for HF can have appropriate testing and intervention.

\section{CONCLUSIONS}

SGLT2 inhibitors have been shown to have a role in the management of HF in patients with T2DM. There is a risk of exacerbation or unmasking of HF when discontinuing SGLT2 inhibitors. To our knowledge, this is the first paper describing the discovery of HF following interruption of SGLT2 inhibitor treatment. The clinician and patient should monitor for signs and symptoms of fluid overload when stopping therapy. Further research into the benefits of a more comprehensive evaluation is needed.

\section{Author disclosures}

The authors report no actual or potential conflicts of interest with regard to this article.

\section{Disclaimer}

The opinions expressed herein are those of the authors and do not necessarily reflect those of Federal Practitioner, Frontline Medical Communications Inc., the US Government, or any of its agencies. This article may discuss unlabeled or investigational use of certain drugs. Please review the complete prescribing information for specific drugs or drug combinations-including indications, contraindications, warnings, and adverse effects-before administering pharmacologic therapy to patients.

\section{References}

1. Thomas MC. Type 2 diabetes and heart failure: challenges and solutions. Curr Cardiol Rev. 2016;12(3):249-255. doi:10.2174/1573403X12666160606120254

2. Patorno E, Pawar A, Franklin J, et al. Empagliflozin and the risk of heart failure hospitalization in routine clinical care: a first analysis from the EMPRISE study. Circulation. 2019;139(25):2822-2830. doi:10.1161/CIRCULATIONAHA.118.039177

3. Packer M. Heart failure: the most important, preventable, and treatable cardiovascular complication of type 2 diabetes. Diabetes Care. 2018;41(1):11-13. doi:10.2337/dci17-0052

4. Thrainsdottir I, Aspelund T, Thorheirsson G, et al. The association between glucose abnormalities and heart failure in the population-based Reykjavík study. Diabetes Care. 2005;28(3):612-616. doi:10.2337/diacare.28.3.612

5. Bell D, Goncalves E. Heart failure in the patient with diabetes: epidemiology, aetiology, prognosis, therapy and the effect of glucose-lowering medications. Diabetes Obes Metab. 2019;21(6):1277-1290. doi:10.1111/dom.13652

6. Nair S, Wilding JPH. Sodium glucose cotransporter 2 Inhibitors as a new treatment for diabetes mellitus. J Clin Endocrinol Metab. 2010;95(1):34-42. doi:10.1210/jc.2009-0473

7. Ali A, Bain S, Hicks D, et al; Improving Diabetes Steering Committee. SGLT2 inhibitors: cardiovascular benefits beyond HbA1c- translating evidence into practice. Diabetes Ther. 2019;10(5):1595-1622. doi:10.1007/s13300-019-0657-8

8. Zinman B, Wanner C, Lachin J, et al; EMPA-REG OUTCOME Investigators. Empagliflozin, cardiovascular outcomes, and mortality in type 2 diabetes. $N$ Engl $J$ Med. 2015;373(22):2117-2128. doi:10.1056/NEJMoa1504720 